\title{
EL TRABAJO AUTÓNOMO - DEPENDIENTE, SUS FORMAS DE INCLUSIÓN EN LA REGULACIÓN PROTECTORA
}

\author{
María del LujÁN Charrutti* \\ Universidad de la República, Uruguay
}

\section{INTRODUCCIÓN}

El trabajo autónomo, es conocido en nuestra realidad jurídica, como es el caso de distribuidores, pequeños artesanos, profesionales, etc. Estos en muchas ocasiones navegan en zonas grises donde no hay una autonomía clara y contundente, mientras que en otras si bien no hay una subordinación jurídica, resulta evidente una total debilidad y exclusividad frente al contratante, propio de un trabajador subordinado pero sin la protección de la normativa laboral, es decir, estamos frente a trabajo autónomo económicamente dependiente.

La doctrina laboral en Uruguay ha planteado en jornadas académicas el rol del Derecho del Trabajo frente a estas inconsistencias, así Raso $^{1}$ se refiere al tema planteando la siguiente interrogante:

“Debe aceptarse la realidad de un mercado de trabajo fragmentado y buscar respuestas jurídicas diferentes a partir de esa diferencialidad, o debemos mantenernos fieles a un derecho del trabajo único para regular lo que ha sido su objetivo tradicional, el trabajo típico? ¿Debemos aceptar un sistema de relaciones laborales con trabajadores diferenciados en sus derechos y obligaciones?".

En Europa donde en algunos países como en España, se aprobó una legislación específica para trabajadores autónomos económicamente dependientes, la doctrina laboral nos alerta sobre la deslaboralización de la prestación a la que ha denominado "huida del derecho del trabajo"2. Se advierte que la estrategia de empleo de la Unión Europea a partir de 2008 contenía modelos de flexiseguridad, definida esta como el otorgar mayor seguridad económica a los trabajadores a cambio de flexibilidad laboral. Se exhortó a los Estados miembros a desarrollar modelos de flexiseguridad en especial en temas como tiempo trabajo, organización del trabajo, contratos flexibles, despidos libres, etc. ${ }^{3}$.

La construcción institucional del Derecho del Trabajo así como su ámbito de aplicación propio ha reposado tradicionalmente sobre una diferenciación técnica de partida, por lo que a la

Docente ayudante grado 1 de Derecho del Trabajo y de la Seguridad Social, Facultad de Derecho Universidad de la República. Magíster en Derecho del Trabajo y de la Seguridad Social

1 Raso Delgue, Juan. “Trabajo autónomo inserto en una hetero organización”, en: VVAA. XXI Jornadas Uruguayas de Derecho del Trabajo y de la Seguridad Social. Uruguay, Montevideo: Fondo de Cultura Universitaria, 2010, p. 120.

2 Baylos Grau, Antonio. Sobre la prestación de trabajo y su laboralidad. Espańa: Publicación Universidad de Castilla La Mancha, 2006.

3 Landa, Juan Pablo. Conferencia sobre Estrategias europeas en materia de empleo dictada en Master en Relaciones Laborales y Diálogo Social en Europa. Espańa, Universidad Complutense de Madrid, 2008. 
realidad social objeto de regulación se refiere: la distinción entre el trabajo asalariado o por cuenta ajena, que integra el territorio propio de la disciplina y cuya articulación jurídica se realiza precisamente a través del contrato de trabajo, y el trabajo autónomo o por cuenta propia, relegado de ese modo contrariamente a una posición extramuros del ámbito de la relación jurídica laboral y de su marco regulador. ${ }^{4}$ Hoy en Uruguay superada la inquietante proliferación de contratos de arrendamientos de servicios ocurridos a partir de 1996 con la promulgación de ley 16713, contratos que en su mayoría ocultaban una relación laboral subordinada y que dio lugar a numerosos juicios en nuestros estrados, comienza a constatarse un nuevo escenario producto de la organización empresarial descentralizada y las necesidades del mercado mundial. Lo que conlleva a distinguir nuevos sujetos en las relaciones laborales: "trabajadores económicamente dependientes" de una empresa no subordinados jurídicamente a la misma, pero sí enmarcados en una posición tan débil y desigual de características similares a las de un trabajador subordinado. Vinculados comercialmente a una empresa en muchas ocasiones con un pacto de exclusividad, no sometidos al poder de dirección de la misma, no subordinados jurídicamente a la misma, pero sí a un control de calidad de cumplimiento del contrato, y con una durabilidad tan extensa que el contratante se encuentra completamente integrado a la organización de la empresa, y cuya extinción le llega a causar un daño material y moral de similar magnitud que el despido mismo. En definitiva como ha señalado Romagnoli cuando hablamos de trabajador autónomo o de trabajo autónomo hablamos de trabajo invisible, de trabajo precario 5 .

El derecho comparado (España) le ha dado una solución legal a la situación del trabajador autónomo económicamente dependiente, estableciendo por vía legislativa, un estatuto para que el derecho del trabajo mantenga, como señala Palomeque: "un cordón umbilical que asegura de modo excepcional una discrecional laboralización parcial de la institución cuando conviene por razones de política social y en aras de una extensión social de los derechos instalados en la relación de trabajo asalariado"'. No obstante el Tribunal Constitucional español" ha establecido límites a esta facultad del poder público, de expulsar del ámbito de aplicación del derecho laboral a prestaciones de trabajo realizadas para empresas en el marco de un proceso de producción de bienes y servicios, marcando su notable contrariedad con el principio de igualdad. Evitando así de esta manera que mediante la ley se pueda llevar a cabo una restricción ilegítima de la noción de trabajador, concepto jurídico político del que por otra parte se deriva el goce de importantes derechos fundamentales como los de libertad sindical y negociación colectiva ${ }^{7}$.

\section{DEL MODELO TRADICIONAL A "LA DESLABORALIZACIÓN DE LA PRESTACIÓN DEL TRABAJO”}

Se pasa por tanto del modelo tradicional de empresa donde la existencia de una única unidad de producción enmarcaba al sindicato en una correlación de fuerzas con un solo empleador, a un modelo descentralizado donde el trabajador sindicalizado debe compartir su área de trabajo

\footnotetext{
4 Palomeque, Manuel Carlos. "El derecho de los trabajadores autónomos a la seguridad social y salud en su trabajo", en: Revista de Derecho Social n40, Albacete, España, p. 11.

5 Romagnoli, Umberto. “Conferencia”, en: Coloquio Italo-Español de Derecho del Trabajo “Globalizzazione, metamorfosi della contrattazione collettiva e tutele dei lavoratori”. Urbino, Italia: Aula Magna de la Facultad de Jurisprudencia, Universidad Carlos Bo, 7 y 8 de octubre de 2011 .

Palomeque, Manuel Carlos, op. cit. (n. 4), p. 13

Baylos Grau, Antonio, “Sobre la prestación de trabajo...”. (n. 2).
} 
con trabajadores autónomos no subordinados al poder de dirección del empleador, los cuales no integrarán por ende la organización sindical. Entonces, el modelo tradicional es aquel donde se encuentra la relación laboral y el contrato de trabajo típico, indefinido, impregnado de cierta estabilidad. Hoy debemos comenzar a estudiar el ámbito subjetivo de aplicación del derecho del trabajo frente a los actuales retos y escenarios que nos presentan las nuevas relaciones laborales.

La laboralización de la prestación es la regla. En la producción de bienes y servicios en que se basa el sistema económico de libre empresa, la aportación de trabajo humano al proceso de producción constituye un elemento decisivo. En los sistemas jurídicos actuales es patente la generalización del trabajo libre por cuenta ajena como fórmula típica de encuadrar la prestación del trabajo humano y productivo para una empresa. Es decir, el trabajo prestado en el ámbito de una organización y dirección de empresa está sometido con carácter general al ordenamiento laboral ${ }^{8}$. En su efecto organizativo como consecuencia del vínculo contractual surge una red de obligaciones entre las partes, esencialmente la obligación de trabajar y remunerar el trabajo, desde luego que este intercambio de trabajo por salario se produce en un ámbito de organización empresarial, es decir, bajo la dirección de un empleador, o lo que es lo mismo de forma subordinada. Lo que significa la inserción en un ámbito de tutelas definidas en lo fundamental por la norma estatal y la negociación colectiva dado que el eje contractual sobre el que se asienta la relación individual, el contrato de trabajo no está concebido de forma que la autonomía individual despliegue plenamente sus efectos en la determinación directa de las condiciones de trabajo y de empleo al punto que este se define como un contrato normado9. La normativa se encarga de proteger a la parte más débil de la relación, el trabajador subordinado. Esta prestación queda comprendida dentro de la competencia de la policía del trabajo, los organismos inspectivos controlarán el cumplimiento de la normativa laboral y de seguridad social, protegiendo al trabajador frente a los incumplimientos del empleador.

No obstante, se presenta una tendencia a la que la doctrina laboral ha denominado "huida del derecho del trabajo". Así la deslaboralización de la prestación de trabajo se define precisamente por su aspecto negativo, es decir por la posibilidad de que las empresas obtengan prestaciones de trabajo para su empleo en el proceso de producción de bienes o servicios que estén organizando sin que la misma sea calificada jurídicamente como sometida al derecho laboral. La deslaboralización implica en consecuencia la exclusión de la prestación de trabajo de que se trate del ámbito de aplicación del ordenamiento jurídico laboral y de la seguridad social sobre el trabajador asalariado o dependiente".

Si bien hay casos en el derecho comparado donde es la misma ley la que excluye del ámbito laboral a determinados casos de trabajadores, el mecanismo más utilizado es el recurso a la autonomía de la voluntad individual de las partes que contratan la prestación de servicios no sometidos a la legislación laboral. Se trata de inscribir la prestación de trabajo en el marco de un contrato no laboral, de naturaleza civil o comercial. La intención y voluntad de las partes regulan directamente el contenido del servicio prestado y las condiciones que rigen éste. Ambas partes son conscientes y en especial la parte económicamente más débil de la naturaleza de su vinculación.

\section{EL IMPACTO EN LAS RELACIONES COLECTIVAS DE TRABAJO}

Los cambios en la organización empresarial, producto de la necesidad de los mercados internacionales de dotar de cierta descentralización a la organización de la empresa de manera

\footnotetext{
Baylos Grau, Antonio. "Prestación del trabajo y partes del contrato", en: Gaeta Lorenzo; Gallardo Moya, Rosario (Coordinadores). Los empresarios complejos: un reto para el Derecho del Trabajo. Espańa, Albacete: Editorial Bomarzo, 2010 , p 67.

9 Baylos Grau, Antonio, “Sobre la prestación de trabajo...”. (n. 2).
} 
de reducir los costes, necesariamente lleva a tener consecuencias en las relaciones colectivas de trabajo. El problema no solo estriba en cómo reconducir al marco del contrato de trabajo la complejidad del empresario, sino que las soluciones pasan también por reequilibrar las representaciones laborales y sindicales que han quedado al margen de los fenómenos de descentralización". La representación sindical ya no es la típica y tradicional que representaba al grupo de trabajadores de la empresa o del sector de actividad en casos de sindicatos de rama, el trabajador debe compartir su espacio de trabajo con un grupo de personas que no se identifican con la organización, así como tampoco revisten la calidad de trabajadores subordinados.

La regulación del trabajo autónomo puede resultar más conflictiva cuando vemos la dimensión colectiva de las relaciones de trabajo prestadas en régimen de autonomía aunque económicamente dependiente. Señala Baylos que se produce un fenómeno de "parasindicalización" de las organizaciones profesionales que representan a los trabajadores autónomos y más en concreto a los económicamente dependientes. Emerge una dimensión colectiva organizativa y regulativa del trabajo autónomo que lleva a la posibilidad de construir una subjetividad colectiva diferenciada de la que significa el sindicato como expresión de un interés colectivo específico del trabajador autónomo".

Por otra parte resulta novedoso en la ley española que regula el Estatuto del Trabajador autónomo económicamente dependiente, la creación de los llamados acuerdos de interés profesional en paralelo a los convenios colectivos, pero establecidos al amparo de disposiciones civilistas entre las asociaciones que representan al trabajador autónomo y las empresas para las que ejecuten su actividad, con una eficacia contractual y no normativa que se limita por ende a las partes firmantes, por tanto no son convenios colectivos sino que son acuerdos con la finalidad de trascender el contrato individual, aunque desempeñan una función de regulación colectiva al establecer las condiciones de modo, tiempo y lugar de ejecución de dicha actividad, así como otras condiciones generales de contratación ${ }^{10}$. Solapadamente también se habla en este instrumento legislativo del derecho de huelga de los trabajadores autónomos (art. 19.2 lit c), al reconocer el derecho a la defensa y tutela colectiva, no obstante habría que repensar tal posibilidad en cuanto a la titularidad del derecho de huelga.

Parte de la doctrina laboral italiana, llega a concluir que la reducción de los costes del trabajo no es el único objetivo perseguido con las externalizaciones, también hay una reducción de los costes que puede llevar el poder sindical ${ }^{11}$. En efecto el poder sindical en los escenarios descentralizados llega a ser menor, consecuentemente la descentralización configura un ahorro significativo para las empresas de gran dimensión y con una gran número de trabajadores sindicalizados, donde se pierden horas de trabajo en casos de conflictos y medidas gremiales.

\section{BREVE REFERENCIA SOBRE EL TRABAJO AUTÓNOMO EN LOS ORDENAMIENTOS LABORALES COMPARADOS}

La Unión Europea, por su parte, en 1986 ya había legislado sobre trabajo autónomo en instrumentos normativos tales como la Directiva 86/613/CEE del Consejo Europeo, relativa a la aplicación del principio de igualdad de trato entre hombres y mujeres que ejerzan una actividad autónoma, incluidas las actividades agrícolas, así como sobre la protección de la maternidad, que da una definición de trabajador autónomo en su artículo 2.a), o en la Recomendación del Consejo

\footnotetext{
10 Palomeque, Manuel Carlos, op. cit. (n. 4), p. 21.

11 LOFREdo, Antonio. "Sobre las transformaciones de la figura del empresario: entre contractualismo y sugerencias institucionalistas", en: Gaeta Lorenzo; Gallardo Moya, Rosario (Coordinadores). Los empresarios complejos: un reto para el Derecho del Trabajo. España, Albacete: Editorial Bomarzo, 2010, p 26.
} 
de 18 de febrero de 2003 relativa a la mejora de la protección de la salud y la seguridad en el trabajo de los trabajadores autónomos. La novedad legislativa como se hizo referencia antes viene de la legislación española que desde 2007 cuenta con un Estatuto del Trabajador Autónomo económicamente dependiente.

Como expresa la exposición de motivos de la mencionada normativa, su regulación obedece a la necesidad de dar cobertura legal a una realidad social: la existencia de un colectivo de trabajadores autónomos que, no obstante su autonomía funcional, desarrollan su actividad con una fuerte y casi exclusiva dependencia económica del empresario o cliente que los contrata. La Ley contempla el supuesto de que este empresario es su principal cliente y de él proviene, al menos, el 75 por ciento de los ingresos del trabajador. La búsqueda de un marco jurídico laboral adaptado al trabajo autónomo se relaciona así con la preocupación de garantizar a estos trabajadores niveles de protección social semejantes a los trabajadores asalariados.

Centrándonos en la normativa importa destacar que la misma exige la concurrencia simultánea de determinados parámetros para ser calificados como trabajadores autónomos económicamente dependientes, como por ejemplo: la condición de no ser empleadores ni contratistas; el desarrollo de su actividad de modo diferenciado en relación con los demás trabajadores al servicio del cliente; la disposición de medios de producción propios (disponer de infraestructura productiva y material propios, necesarios para el ejercicio de la actividad e independientes de los de su cliente, cuando en dicha actividad sean relevantes económicamente); la disposición de una organización productiva propia, sin perjuicio de las indicaciones técnicas que pudiese recibir de su cliente. Marcando claramente que su actividad profesional debe ser desarrollada con criterios organizativos propios no sometido a ninguna dirección, no existiendo sometimiento alguno al poder de dirección del contratante, salvo las indicaciones técnicas producto de la organización en la cual está inserta el cliente. Por otra parte se establecen artículos que refieren a la duración del contrato, la interrupción anual de la actividad, el régimen de descanso semanal y festivo, la jornada y su distribución semanal, la realización de la actividad por un tiempo superior al pactado, el horario de actividad y su adaptación a las necesidades de conciliación de la vida personal, familiar y profesional del trabajador autónomo, de efectividad de la protección contra la violencia de género, la extinción del contrato, la interrupción o suspensión de la actividad por parte del trabajador, los acuerdos de interés profesional y la protección de la seguridad y salud en el trabajo.

\section{EL TRABAJO AUTÓNOMO EN URUGUAY}

En Uruguay el modelo flexible se instala a partir de 1990 cuando dejan de convocarse los Consejos de Salarios (órganos tripartitos para la fijación de salarios mínimos, categorías y condiciones de trabajo), la ausencia de la negociación colectiva hizo que los trabajadores no contaran con un ámbito colectivo para dirimir sus conflictos y consecuentemente disminuyeran sus garantías laborales, lo que implicó una creciente pérdida del salario real.

Sumado a ello, se sancionan dos leyes que tuvieron un efecto nocivo para el mercado de trabajo y las condiciones laborales de los trabajadores. La primera fue la Ley No 16.906 de 1996 sobre caducidad de los créditos laborales, la cual redujo sensiblemente el plazo de reclamaciones laborales, pasando de diez años a dos fomentando así el incumplimiento laboral. La segunda ley inspirada en la flexibilidad laboral fue la ley 16713 , norma sobre reforma del sistema jubilatorio que introduce un régimen mixto (capitalización individual y solidaridad intergeneracional), esta norma incluyó en el art. 178 una regulación específica sobre el régimen de contratación con empresas unipersonales. Este 
artículo tuvo un gran impacto en el mercado laboral, lo que llevó a que se expandiera la figura del trabajador autónomo, pero con un efecto negativo para el derecho laboral dado que las empresas no siempre utilizaban esta figura para su verdadero fin (empresa unipersonal), sino para reducir costos laborales contratando trabajadores subordinados bajo el rótulo de arrendamientos de servicios. Esto llevó a que fueran numerosos los casos que se presentaron ante la justicia solicitando la aplicación del principio de primacía de la realidad. Los resultados en su gran mayoría fueron favorables al trabajador, desenmascarando de esta manera verdaderas relaciones laborales. Este modelo flexible instalado en Uruguay tuvo su réplica en el resto de América Latina.

A partir del año 2005 al convocarse nuevamente los Consejos de Salarios y ampliando su espectro incluyendo al sector rural y doméstico, trajo como consecuencia inmediatamente el desplazamiento de aquel modelo flexibilizador. Aumenta la tasa de afiliación sindical, se pontencializa la negociación colectiva y pasa a tener un rol protagónico el Sindicato. Se sancionaron hasta la fecha numerosas leyes protectoras y decretos, siendo de mayor trascendencia e importancia la ley 17940 sobre fuero sindical, la cual establece la nulidad de los actos discriminatorios como el despido. Todos estos cambios han fortalecido las relaciones laborales, bajando las tasas de desempleo, provocando un crecimiento importante del salario real, y ampliando cada vez más la protección del trabajador subordinado. Se refleja por tanto una coyuntura distinta a la que está viviendo el modelo implantado por la Unión Europea. En tal sentido, se ha vuelto frecuente que la justicia laboral uruguaya ampare a trabajadores autónomos que reclaman relación de dependencia, apoyados en la Recomendación $\mathrm{N}^{\circ} 198$ de la OIT sobre relación de trabajo. También contribuye a ello, la derogación del citado art. 178 de la ley 16713 por ley 18.783 de julio de 2011, eliminando así las formas de incentivo y fomento de contratación de obra y servicio.

No obstante lo expuesto queda un sector de trabajadores autónomos, los verdaderos autónomos (aquellos no dependientes), no subordinados a la potestad de dirección de un empleador, pero sí dependientes económicamente a estos, estos trabajadores deberían ser protegidos por el derecho del trabajo.

\section{POSIBLES FORMAS DE INCLUSIÓN EN LA REGULACIÓN PROTECTORA}

La doctrina laboral uruguaya viene contemplando esta situación. En esta línea Rodríguez Azcue, focalizando su estudio como el resto de la doctrina (Barbagelata, Mantero, Raso, Ermida entre otros), en lo dispuesto en nuestra Constitución en el art. 53, el cual dice "el trabajo está bajo la protección de la ley", entiende que esta no limita su alcance al trabajo dependiente, sino que la protección abarca a todo tipo de trabajo, tanto al subordinado como al autónomo. En consecuencia, la doctrina sostiene que en materia de derechos laborales se constata la inexistencia de una normativa mínima que regule los derechos básicos a este sector, mientras que en materia de seguridad social se aprecian algunos avances (protección de riesgos de invalidez, vejez, sobrevivencia y prestaciones por maternidad). Otros riesgos no son protegidos, como por ejemplo el desempleo, los accidentes de trabajo, las enfermedades profesionales. Esta visualización ha conducido a considerar a un amplio sector de la doctrina en Uruguay que podría plantearse la necesidad de un estatuto protector del trabajador autónomo, no como puerta de salida del Derecho del Trabajo, sino como expansión de éste ${ }^{12}$.

\footnotetext{
Rodríguez Azcue, Álvaro. "Empresas Unipersonales: ¿Modificación o extinción?, en: en: VVAA. XXI Jornadas Uruguayas de Derecho del Trabajo y de la Seguridad Social. Uruguay, Montevideo: Fondo de Cultura Universitaria, 2010, p. 114.
} 
No obstante, las desventajas de la sanción de una ley ya han sido expuestas por la doctrina española al alertarnos sobre denominada "delaboralización de la prestación de trabajo". Cabe tener presente también lo expuesto por el Tribunal Constitucional español, que plantea la constitucionalidad de una decisión del legislador de excluir a determinadas prestaciones del amparo de la protección laboral. Dándole paso a la autonomía de la voluntad los actores sociales serían los sujetos mayormente legitimados para limitar estas nuevas formas de empleo, como se ha hecho en algunas ocasiones en Uruguay con los procesos de tercerización en la industria de la alimentación. Así, en la tercera ronda de Consejos de Salarios (acuerdo de Consejo de Salarios del 1ro de noviembre de 2008 Grupo 1 de actividad Procesamiento y conservación de alimentos y bebidas (subgrupo Dulce y Panificadoras), se incluyó una cláusula estableciendo algunas limitantes al poder de organización de la empresa. La cláusula novena del referido acuerdo señala:

“Tercerizaciones: "Solamente se admitirá la tercerización de aquellos sectores, tareas, funciones o servicios que no estén directamente vinculados con la producción, exceptuándose solo aquellas situaciones de incremento temporal de trabajo. Se entenderá por incremento todos los aumentos de producción que excedan los niveles estándar o normales de la actividad y/o que puedan ser realizados con la plantilla fija o estable del personal: "temporal “ significa que está relacionado por un período de tiempo determinado, que marca la excepcionalidad de la actividad frente al principio general de la continuidad del derecho del trabajo. De acuerdo al marco normativo vigente y criterios interpretativos de doctrinas y jurisprudencia, dichos plazos excepcionales deberán ser previamente establecidos y documentarse mediante los contratos de trabajo a término que corresponda”.

Un marco jurídico adecuado que evite la figura del falso autónomo, la contratación de autónomos para el sector de producción, la sustitución de puestos de trabajo subordinado por trabajadores autónomos, o la contratación de autónomos frente a medidas sindicales como el caso de "contratación de rompe huelgas", evitaría situaciones conflictivas y a su vez dotaría de cierta protección al trabajador autónomo económicamente dependiente. Por tanto, puede ser el convenio colectivo el instrumento necesario para canalizar esta construcción como limitativa del poder del empresario, como orientadora de las relaciones laborales. No solo importa integrar la declaración constitucional del art. 53 "el trabajo está bajo la protección de la ley" y proyectarla hacia el sistema de relaciones laborales a través de todos los operadores jurídicos, sino que lo importante es que por la vía del diálogo social se desarrollen y extiendan los derechos laborales.

\section{BIBLIOGRAFÍA}

Baylos Grau, Antonio. "Prestación del trabajo y partes del contrato”, en: GaEta Lorenzo; Gallardo Moya, Rosario (Coordinadores). Los empresarios complejos: un reto para el Derecho del Trabajo. Espańa, Albacete: Editorial Bomarzo, 2010.

Baylos Grau, Antonio. Sobre la prestación de trabajo y su laboralidad. España: Publicación Universidad de Castilla La Mancha, 2006.

LANDA, Juan Pablo. Conferencia sobre Estrategias europeas en materia de empleo dictada en Master en Relaciones Laborales y Diálogo Social en Europa. España, Universidad Complutense de Madrid, 2008.

Lofredo, Antonio. "Sobre las transformaciones de la figura del empresario: entre contractualismo y sugerencias institucionalistas", en: Gaeta, Lorenzo; Gallardo Moya, Rosario (Coordinadores). Los empresarios complejos: un reto para el Derecho del Trabajo. España, Albacete: Editorial Bomarzo, 2010. 
Palomeque, Manuel Carlos. "El derecho de los trabajadores autónomos a la seguridad social y salud en su trabajo", en: Revista de Derecho Social n0, Albacete, Espańa.

PÉRez Rey, Joaquín. "El empresario complejo y la necesidad de un derecho del trabajo insolente", en: GaETA, Lorenzo; Gallardo Moya, Rosario (Coordinadores). Los empresarios complejos: un reto para el Derecho del Trabajo. España, Albacete: Editorial Bomarzo, 2010.

Raso Delgue, Juan. “Trabajo autónomo inserto en una hetero organización”, en: VVAA. XXI Jornadas Uruguayas de Derecho del Trabajo y de la Seguridad Social. Uruguay, Montevideo: Fondo de Cultura Universitaria, 2010.

Rodríguez Azcue, Álvaro. "Empresas Unipersonales: ¿Modificación o extinción?, en: en: VVAA. XXI Jornadas Uruguayas de Derecho del Trabajo y de la Seguridad Social. Uruguay, Montevideo: Fondo de Cultura Universitaria, 2010.

Romagnoli, Umberto. "Conferencia”, en: Coloquio Italo-Español de Derecho del Trabajo "Globalizzazione, metamorfosi della contrattazione collettiva e tutele dei lavoratori”. Urbino, Italia: Aula Magna de la Facultad de Jurisprudencia, Universidad Carlos Bo, 7 y 8 de octubre de 2011. 